\title{
Compromiso respiratorio en mucopolisacaridosis
}

\author{
Dr. Jury Hernández'
}

1 Neumólogo Pediatra. Complejo Asistencial Dr. Víctor Ríos Ruiz. Jefe Unidad de Paciente Crítico. Los Ángeles, Chile.

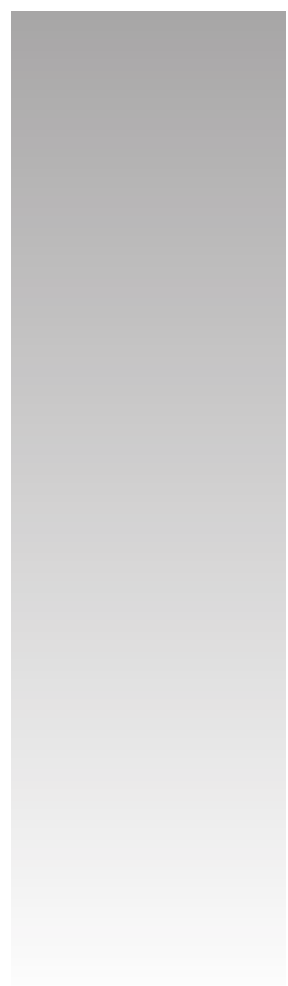

\section{RESPIRATORY MANIFESTATIONS IN MUCOPOLYSACCHARIDOSIS}

Mucopolysaccharidosis (MPS) are part of the so-called lysosomal diseases, in which the deposit of different glycosaminoglycans, depending on the enzyme deficit, generates multi-systemic manifestations, being the respiratory system one of the most affected and associated with significant morbidity and mortality. Different types of MPS show a variable degree of organ compromise even from the early stages of life: obstruction of the upper airway of varying degree, persistent rhinorrhea, otitis media, obstructive pathology of the peripheral airway, pneumonias or other infections associated with a poor mucociliary drainage are the main manifestations presented by patients. The compromise of the neurological and musculoskeletal system also brings the compromise of the respiratory pump. From that perspective the approach must be multidisciplinary, since there are several organs and systems involved. Current therapy is directed to replace the deficient enzyme but it's available only for some of them, which delays the progression of the disease but does not stop it, even more so there is no effect on the central nervous system, being the cognitive compromise inevitable. Bone marrow transplant is a therapy not exempt of complications, but capable of changing the progression of the disease in its early stages. Therapeutic approach is based on support measures and treatment of concurrent complications, both of which will be discussed in the following article.

Key words: Mucopolysaccharidosis, respiratory compromise, storage disease, multisystemic, morbidity.

\section{RESUMEN}

Las Mucopoliscaridosis (MPS) son parte de las denominadas enfermedades lisosomales. El depósito de los distintos glicosaminoglicanos comprometidos, dependiendo del déficit enzimático, genera manifestaciones multisistémicas, en donde el sistema respiratorio es uno de los principales afectados y que se asocia con morbilidad y mortalidad significativa. Los diferentes tipos de MPS presentan un grado variable de compromiso desde etapas precoces de la vida, síntomas de obstrucción de vía aérea superior de grado variable, rinorrea persistente, otitis media, patología obstructiva de vía aérea periférica, neumonías o infecciones asociadas a un mal drenaje mucociliar son las principales manifestaciones que los pacientes presentan. El compromiso neurológico y musculo esquelético, trae consigo además el compromiso de la bomba respiratoria. Desde esa perspectiva el enfoque debe ser multidisciplinario, ya que el compromiso abarca varios órganos y sistemas. Las actuales terapias están dirigidas a reemplazar la enzima deficitaria, disponibles sólo para algunas de ellas, esto trae consigo el retardo de la evolución de la enfermedad pero no lo evita, considerando que más aun no tiene ningún efecto sobre el sistema nervioso central, por lo que el compromiso cognitivo es inevitable. El trasplante de médula es una terapia no exenta de complicaciones, pero que es capaz de cambiar la progresión de la enfermedad en las etapas precoces de ella. El enfoque terapéutico se basa en terapia de sostén y el manejo de las distintas complicaciones que se van dando, siendo éstos los ejes del siguiente artículo.

Palabras clave: Mucopolisacaridosis, compromiso respiratorio, enfermedad de deposito, multisistémico, morbilidad.

\section{INTRODUCCIÓN}

La mucopolisacaridosis (MPS) es una enfermedad que esta incluida dentro de las enfermedades lisosomales (enfermedades de depósito), considerada por mucho tiempo como una enfermedad rara o huérfana, dada su baja incidencia y alta

Correspondencia: Dr. Jury Hernández.

Neumólogo Pediatra. Complejo Asistencial Dr. Víctor Ríos Ruiz.

Jefe Unidad de Paciente Crítico, Los Ángeles. Chile.

E-mail: jury.hernandez@ssbiobio.cl

ISSN 07|8-332I Derechos reservados. morbi-mortalidad ${ }^{(1-3)}$. Se trata de un trastorno genético, en donde existe un déficit enzimático específico encargado del metabolismo de los glicosaminoglicanos (GAGS). Los principales GAGS son el dermatán, heparán, keratán y condroitín sulfato y por ende, su déficit desencadena la acumulación de sustratos a nivel intracelular, en los tejidos u órganos donde estos se encuentran mas representados ${ }^{(4-7)}$. Esto trae consigo que las manifestaciones y compromiso sea multisistémico, involucrando, el sistema músculo esquelético, nervioso, respiratorio, digestivo, renal, cardiovascular, etc $c^{(8-1)}$.

Las MPS dependiendo del déficit enzimático se han sido dividido en 7 tipos, asociados a II déficit enzimáticos específicos $^{(1,3)}$. Según el tipo de MPS el compromiso respiratorio 
puede ser precoz y más severo, afectando desde la vía aérea superior, trastornos de la esfera otorrinolaringológica(4), alteraciones a nivel de la vía aérea inferior, compromiso del parénquima pulmonar como también de la bomba respiratoria (compromiso neuromuscular como de parrilla costal), y además disturbios del sueño, siendo el compromiso respiratorio una de las principales causas de morbilidad y mortalidad en este tipo de pacientes ${ }^{(2)}$.

La terapia de reemplazo enzimática (TRE) disponible para algunos tipos de MPS y el trasplante de médula ósea (en edades precoces de la vida), muchos de estos pacientes tienen un pronóstico de vida favorable ${ }^{(12)}$. Analizaremos los principales aspectos desde el punto de vista del sistema respiratorio que compromete a la mucopolisacaridosis

\section{ASPECTOS GENERALES}

Las MPS se consideran un grupo amplio de trastornos genéticos asociados al déficit de enzimas lisosomales encargadas del metabolismo de los GAGS. Los GAGS se encuentran en la superficie celular como en la matriz extracelular. Las manifestaciones van desde cuadros leves hasta formas muy severas (dependiendo del tipo de trastorno y momento diagnóstico). Actualmente existen diversas guías clínicas para establecer el diagnóstico de un gran número de ellas ${ }^{(5,6)}$. Las MPS se dividen en siete tipos (Tabla I).

La MPS tipo I o síndrome de Hurler es una enfermedad autosómica recesiva y dependiendo del tipo de mutación, existen las formas severas (síndrome de Hurler), la forma atenuada (síndrome de Hurler-Scheie) y la forma leve (enfermedad de Scheie). Se debe al déficit enzimático de la $\alpha$-Liduronidasa con la consecuente acumulación de dermatán y heparán sulfato. La incidencia del síndrome de Hurler es I : I 00.000 recien nacido (RN) vivos y del síndrome de Scheie de 1:800.000 RN vivos ${ }^{(6-13)}$.

La MPS tipo II o síndrome de Hunter, se debe al déficit enzimático de la irunidato-2-sulfatasa. A diferencia de las otras MPS, este es un trastorno que afecta principalmente a hombres jóvenes, ya que tiene una herencia ligada al cromosoma $X$ y sólo hace algunos años se han descrito formas que afectan a mujeres. Su incidencia es 0,3 I a 0,7I por cada 100.000 $\mathrm{RN}$ vivos. Al igual que la MPS tipo I tiene manifestaciones multisistémicas, en donde el sistema respiratorio es uno de los principalmente afectados con obstrucción moderada a severa de la vía aérea superior como también la afectación a nivel de sistema nervioso central y compromiso cardíaco(4-14).

La MPS tipo III o síndrome de Sanfilippo, se divide en 4 tipos, dependiendo del tipo de déficit enzimático que presenta, afectando el metabolismo del heparán sulfato, también se trata de une enfermedad autosómica recesiva, en la que sus síntomas se centran fundamentalmente a nivel de sistema nervioso central. Se manifiesta con características faciales especificas, además de retraso del desarrollo psicomotor, alteraciones cognitivas, ciclo de sueño vigilia, agresividad, hiperactividad y además conducta autista(15).

La MPS tipo IV o enfermedad de Morquio se produce por un defecto enzimático en el metabolismo del keratán sulfato. Las principales manifestaciones se dan en el sistema osteomuscular (depósito en hueso y tejido cartilaginoso), cursan son disostosis marcada, además compromiso articular progresivo, talla baja o enanismo (diagnóstico diferencial con las displasias óseas), compromiso a nivel cardiaco (válvulas) y ocular, subluxación de columna cervical, además de infecciones respiratorias recurrentes y compromiso de parrilla costal y compromiso de la bomba respiratoria(16).

Tabla I. Clasificación de las MPS y compromiso respiratorio

\begin{tabular}{|c|c|c|c|c|c|c|}
\hline Tipo & Epónimo & Enzima deficitaria & $\begin{array}{c}\text { GAGS } \\
\text { comprometido }\end{array}$ & $\begin{array}{l}\text { Obstrucción } \\
\text { de VAS }\end{array}$ & $\begin{array}{c}\text { Obstrucción } \\
\text { de VAl }\end{array}$ & $\begin{array}{c}\text { Compromiso } \\
\text { restrictivo }\end{array}$ \\
\hline I & $\begin{array}{l}\text { a) Hurler } \\
\text { b) Hurler- Scheie } \\
\text { c) Scheie }\end{array}$ & $\alpha$-L-Iduronidasa & $\begin{array}{l}\text { Dermatán y Heparán } \\
\text { sulfato }\end{array}$ & +++ & +++ & +++ \\
\hline$\|$ & Hunter & Iduronato 2- sulfatasa & $\begin{array}{l}\text { Dermatán y Heparán } \\
\text { sulfato }\end{array}$ & +++ & +++ & ++ \\
\hline III & Sanfilippo (A-D) & $\begin{array}{l}\text { A) Heparan Sulfamidasa } \\
\text { B) N-Acetyl-Glucosaminidasa } \\
\text { C) Acetyl-CoA: alpha-glucosamindea- } \\
\text { cetyltransferasa } \\
\text { D) N-acetylglucosamine 6-sulfatasa }\end{array}$ & $\begin{array}{l}\text { Heparán Sulfato } \\
\text { (todos los subtipos) }\end{array}$ & mínimo & mínimo & mínimo \\
\hline IV & Morquio (A-B) & $\begin{array}{l}\text { A) Galactose-6-sulfato sulfatasa } \\
\text { B) Beta-galactosidasa }\end{array}$ & $\begin{array}{l}\text { A) Keratán Sulfato y } \\
\text { condroitín } 6 \text { sulfato } \\
\text { B) Keratán Sulfato }\end{array}$ & ++ & + & +++ \\
\hline $\mathrm{Vl}$ & Maroteaux-Lamy & $\mathrm{N}$-acetyl-galactosamine-4-sulfatasa & Dermatán Sulfato & +++ & +++ & ++ \\
\hline VII & Sly & Beta-glucuronidasa & $\begin{array}{l}\text { Dermatán sulfato y } \\
\text { Heparán sulfato }\end{array}$ & +++ & +++ & ++ \\
\hline IX & Hyaluronidasa-I & Hyaluronan & & & & \\
\hline
\end{tabular}

VAO: Vía aérea superior; VAl: Vía aérea inferior; GAGS: Glicosaminoglicanos. 
La MPS tipo VI, o síndrome de Maroteux-Lamy, se produce por el defecto enzimático en la $\mathrm{N}$-acetil galactosaminasulfato. Se caracteriza por la facie típica, opacidad corneal progresiva, compromiso osteo articular, alteraciones a nivel de columna con cifoscoliosis, disfunción atloaxoidea, estenosis de agujero magno, hidrocefalia, compromiso respiratorio, de múltiples causas que analizaremos mas adelantes. Es también relevante el compromiso de válvulas cardiacas, que puede generar disfunción miocárdica(4-7).

Otras 2 formas mucho menos frecuentes, son la MPS tipo VII por déficit de b-glucoronidasa y la MPS tipo IX debido al déficit de hialuronidasa, en donde se han descrito escasos casos hasta la fecha. En Chile, ha sido la agrupación de padres de pacientes con enfermedades lisosomales, conocidos con la sigla FELCH(17), quienes han llevado registro de los pacientes con MPS. Actualmente en Chile existen 53 pacientes con MPS, que se distribuyen en: 14 tipo I, 13 tipo II, 7 tipo III, I 6 tipo IV y I I tipo VI; han fallecido un total de 12 pacientes hasta la fecha (3 tipo I, 6 tipo II, I tipo III y 2 tipo VI).

En nuestro Servicio de Salud, que consta con una población infantil aproximada de 100.000 habitantes menor de 15 años, contamos actualmente con 2 pacientes con MPS tipo I (Figura I), ambos con formas severas; 3 pacientes con MPS tipo III (2 de ellos hermanos) y una paciente con MPS tipo $\mathrm{VI}$ (Figura 2), de las cuales 2 de ellas están con terapia de reemplazo enzimática, a pesar de lo cual, la progresión de la enfermedad se ha hecho evidente en los años.

\section{MANIFESTACIONES RESPIRATORIAS}

\section{Compromiso nasal y auditivo}

Las manifestaciones a nivel otorrinolaringológicas son universales, siendo muchas de ellas las iniciales de la enfermedad. Los pacientes con MPS en general tienen rinorrea nasal crónica o persistente, además de secreciones desde la vía aérea superior e inferior explicado por obstrucción mecánica dado infiltración de la mucosa, por un proceso inflamatorio persistente o por la calidad de las secreciones desde la glándulas de mucosa y submucosa en donde los principales GAGS son

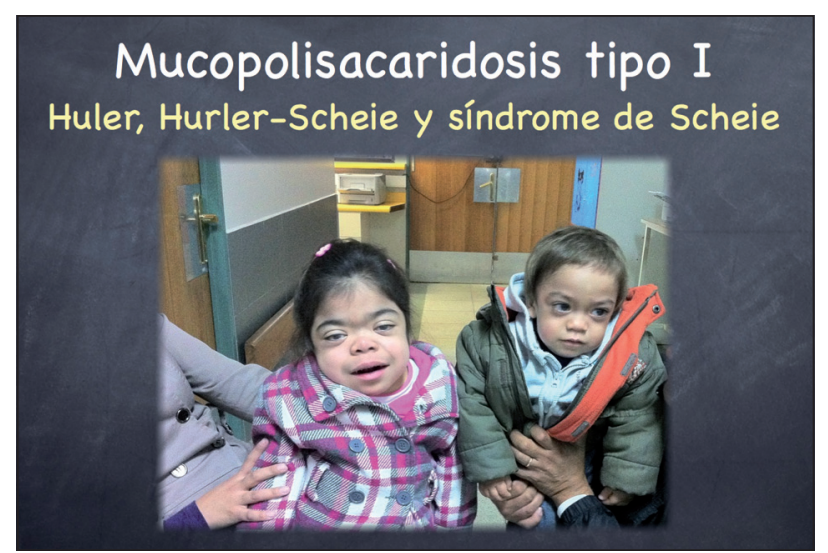

Figura I. Pacientes con MPS I. Los Angeles.

dermatán y keratán sulfato(5). Los pacientes con MPS tipo I y II, frecuentemente cursan con otitis media con efusión u otitis media crónica, con riesgo de infecciones recurrentes por lo que la instalación de tubos de ventilación es una condición frecuente en este grupo de pacientes. El compromiso auditivo esta dado por una hipoacusia de conducción y/o sensorio neural, afectando al 50-80\% de los pacientes con MPS tipo I, II ○ VI, según las distintas series publicadas ${ }^{(18)}$. La evaluación con impedanciometría, audiometría, potenciales evocados y nasofibroscopía es importante en todo paciente con MPS con el fin de evaluar el compromiso funcional desde el punto vista otológico y en donde el retraso lingüístico no sólo puede estar influenciado por la hipoacusia sino que también por el retraso mental de algunas de ellas. Muchos de los pacientes requerirán inserción de tubos de ventilación para mejorar la hipoacusia de conducción por disfunción tubárica, secundaria a la infiltración de GAGS a nivel de la mucosa respiratoria como también el compromiso de membrana timpánica y la cadena oscicular ${ }^{(19)}$. La hipertrofia adenotonsilar de los pacientes es una constante siendo un factor que contribuye a la hipoacusia y los síntomas de obstrucción de vía aérea superior con apneas secundarias, asociado a infiltración con material PAS positivo.
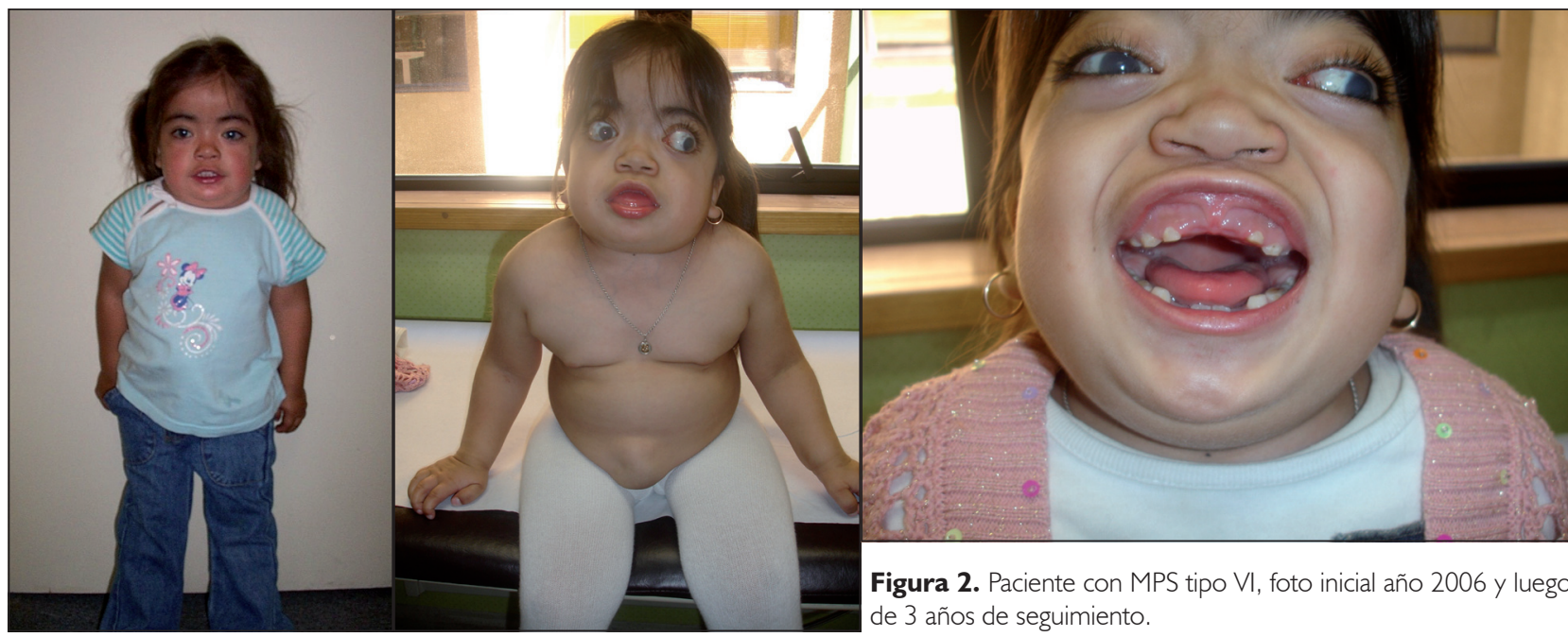

Figura 2. Paciente con MPS tipo VI, foto inicial año 2006 y luego de 3 años de seguimiento. 

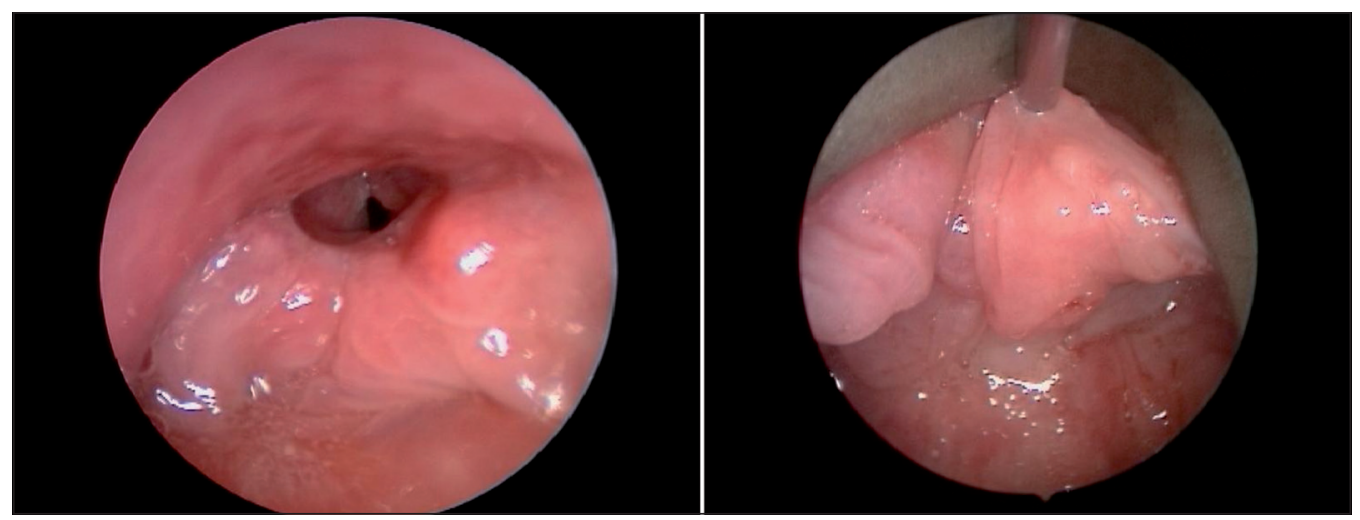

Figura 3. Compromiso laríngeo en paciente con MPS tipo I, forma severa.

\section{Obstrucción de la vía aérea superior}

La MPS se caracteriza en general con rasgos faciales, siendo una vez que avanza la enfermedad cada vez mas evidentes: Pelo ralo, cara tosca, piel gruesa, puente nasal plano, crestas supra orbitarias prominentes, mejillas prominente, macroglosia con una relación desfavorable del tamaño de la lengua en relación cavidad oral, con una consecuente obstrucción de la vía aérea especialmente en decúbito y en momentos que impera mas hipotonía de la musculatura faríngea o como ocurre en el sueño, con apneas obstructivas secundarias ${ }^{(20-21)}$. La infiltración de los GAGS se hace manifiesto en gran parte de las estructuras de la vía aérea como la laringe (Figura 3) al igual que adenoides y amígdalas palatinas o amígdala lingual ${ }^{(3)}$. Estudios con tomografía axial computada hacen evidente el compromiso de la vía aérea superior de estos pacientes, desde el compromiso cordal y disminución del área de sección de la tráquea cuando se compara en todas las edades con pacientes sin MPS(22). La infiltración de la laringe de los pacientes se hace de manifiesto con presencia frecuente de estridor, voz disfónica característica, principalmente en las MPS tipo I, Il y VI, que se corrobora con los estudios de imágenes (nasofibroscópicos y fibrobroncoscópicos), estudios histológicos y radiológicos en estos pacientes ${ }^{(23)}$.

Los pacientes que cursan con obstrucción severa de la vía aérea superior, pueden verse beneficiados transitoriamente con cirugía de adenotonsilectomía, considerando que la infiltración con GAGS puede recidivar; además se debe considerar el riesgo de complicaciones de la cirugía como es el riesgo de sangrado y la falla en extubación considerando pacientes con vía aérea difícil, análisis o consideraciones que haremos mención especial en esta revisión. Es sabido que hoy una de las mejores alternativas para el tratamiento de las apneas obstructivas es el uso de generadores de flujo, con CPAP (presión positiva continua de la vía aérea) o BiPAP (presión binivelada de la vía aérea), dependiendo del grado y severidad de las mismas y si se asocia a apneas de origen central, situación que es frecuente en los pacientes con MPS, considerando las complicaciones neurológicas de los pacientes (hidrocefalia, compromiso o estenosis de agujero magno por infiltración de la duramadre, inestabilidad de la columna cervical) ${ }^{(20)}$. Los eventos de obstrucción pueden ser tan severos como generar hipoxia secundaria y consecuentemente hipertensión pulmonar secundaria ${ }^{(24)}$. En los casos que no se pueda instalar un soporte ventilatorio, a través de una interface nasal, o en eventos de urgencia relacionados a una falla en el destete después de alguna cirugía, la traqueotomía debe ser planteada como una buena alternativa, considerando que es un procedimiento no libre de complicaciones: aspiración frecuente, obstrucción de la cánula por secreciones o tapones de mocos con material PAS +, obstrucción distal del lumen de cánula por tejido o granulomas, estenosis distal a cánula de traqueostomía, etc ${ }^{(25)}$.

\section{Obstrucción de la vía aérea inferior}

Los pacientes con MPS en general cursan con inflamación importante de la vía aérea, además de disminución del lumen de ella asociado a infiltración de la mucosa por los GAGS, infiltración que hace que el lumen sea irregular, sumado a lo anterior es la hipersecreción bronquial, además de disminución del drenaje mucociliar que estos pacientes presenten, esto ha sido evaluado en base a estudios radiológicos por tomografía axial computada con reconstrucción, además de evaluación fibrobroncoscópica que corrobora lo anterior, además de poder evaluar colapso dinámico de la vía aérea (traqueobroncomalacia) situación bastante frecuente en los pacientes con MPS, hallazgo muy importante al momento de procedimientos quirúrgicos y anestesia de los pacientes, razón por la que es importante una evaluación objetiva previo a la realización del acto anestésico con el fin de definir la mejor técnica con el fin de evitar complicaciones ${ }^{(22,23,26)}$.

Es bastante frecuente el uso de terapia broncodilatadora, del tipo beta agonistas y anticolinérgicos, considerando la hipersecreción bronquial; al igual que los corticoides sistémicos e inhalados como terapia antiinflamatoria, semejando la fisiopatología de asma bronquial. No existen estudios que hayan evaluado la respuesta clínica y de laboratorio en base a cambios en volúmenes o flujos espiratorios con espirometria u otras técnicas diagnósticas. Un reporte de función pulmonar realizado en un paciente con MPS tipo II fue evidente el patrón obstructivo asociado a una capacidad vital forzada (CVF) disminuida, con un deterioro en el tiempo (27). Los mismos hallazgos fueron observados en pacientes con MPS tipo IV o enfermedad de Morquio(28). Estos mismos parámetros han servido actualmente para poder evaluar la respuesta a la TRE como una forma de objetivar la mejoría del paciente. Un estudio multicéntrico realizado en pacientes con MPS tipo VI, 
evaluando la respuesta a TRE con medición de VEF, y CVF previo y posterior a la terapia, observándose una mejoría del 14\% en ambos parámetros a las 72 semanas de terapia y 17\% para CVF y I I \% para VEF, a las 96 semanas posterior al inicio de tratamiento. No se conoce si esto es debido a una disminución en la inflamación o infiltración de la vía aérea, como mejoría de los volúmenes pulmonares al disminuir la visceromegalia que presentan, mejorando así los volúmenes y capacidades pulmonares o la asociación de una mejoría propia del crecimiento corporal de los niños ${ }^{(29)}$.

Una de las complicaciones mas frecuentes asociadas a los pacientes con MPS, de etiología multifactorial es la presencia de atelectasias, muchas de ellas de difícil manejo que requieren manejo kinésico, terapia broncodilatadora, o uso de lavado bronco alveolar con fibrobroncoscopía, con el fin de poder lograr una rápida re expansión pulmonar, terapia que se sugiere que se haga con un equipo multidisciplinario, y con experiencia en el manejo de vía aérea difícil, es fundamental el soporte con anestesistas que manejen uso de mascara laríngea, ya que es uno de las principales herramientas utilizadas en pacientes con MPS no solo para la realización de procedimientos, sino que también para intubaciones difíciles.

Se adjuntan 2 imágenes de radiografías de pacientes con MPS VI y I que cursaron con atelectasias importantes y que determinaron manejo intensivo (Figura 4). No se debe olvidar que estos pacientes son susceptibles de infectarse por agentes que pueden generar una inflamación mayor de su vía aérea, como son virus respiratorio sincicial, metaneumovirus humano $u$ otros agentes que generen cuadros que determinen un deterioro agudo que implique caer en insuficiencia respiratoria y necesidad de ventilación mecánica(30). Existen pacientes que han cursado con cuadros de insuficiencia respiratoria y falla durante la extubación, habiendo sido necesario la traqueostomía, con los riesgos asociados. También se han publicado reporte de casos que han requerido del uso de stent traqueales y/o bronquiales ${ }^{(3 l)}$.

\section{Enfermedad pulmonar restrictiva}

Los pacientes con MPS que principalmente presentan alteraciones de la caja torácica son aquellos que presentan compromiso esquelético más severo: MPS tipo I, II, IV y VI; ya sea por las deformidades propias asociadas en las costillas (articulaciones condrocostales rígidas), costillas mas horizontales y escoliosis, xifosis, xifoescoliosis o pectum carinatum. Se suma a los factores anatómicos estructurales de la parrilla costal, la presencia de hepatoesplenomegalia, determinando la disminución de la excursión torácica y reducción de la capacidad residual funcional.

La evaluación de la enfermedad pulmonar de los pacientes, se puede realizar con espirometría, en donde el patrón restrictivo es la regla con o sin componente obstructivo, dependiendo del grado de cooperación (limitaciones sensoriales o mentales). También es posible evaluar los volúmenes pulmonares mediante pletismografía o por técnicas de dilución, experiencia antigua en pacientes con MPS tipo IV quienes mostraron el compromiso restrictivo de su enfermedad y el riesgo de morbimortalidad pulmonar asociada ${ }^{(28)}$. Estudios con oscilometría de impulso y movimientos toracoabdominales realizado en pacientes con displasia esquelética, donde

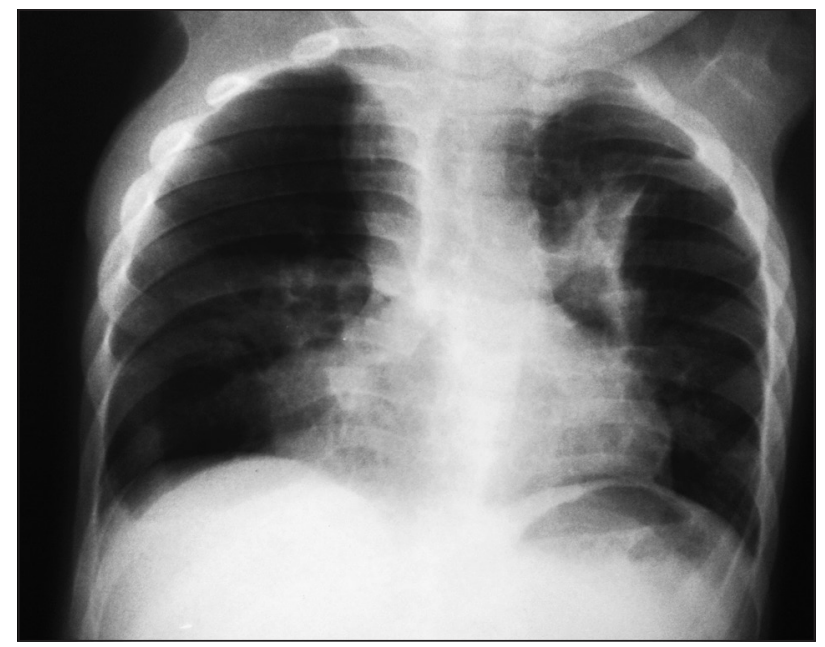

Figura 4. Radiografía de Tórax Ap paciente con MPS e infección por hMNV.

se encontraban 4 pacientes con MPS tipo IV revelaron la disminución en la XRS a los $5 \mathrm{~Hz}$ y aumento en $\operatorname{Rr}^{(32)}$. El rol de la terapia de reemplazo enzimática en MPS ha tenido un rol fundamental en la mejoría de la función pulmonar en pacientes con MPS I, II y tipo VI, mejorando los parámetros de CVF luego de 26 y 96 semanas de terapia de reemplazo enzimático respectivamente, al igual que mejoría en el test de marcha de 6 minutos y ventilación voluntaria máxima de los pacientes tratados con MPS tipo VI. La terapia de reemplazo enzimática, no es capaz de evitar el compromiso de articulaciones y a nivel de parilla costal razones por la cual el soporte médico es fundamental. ${ }^{29-33-34-35}$

\section{TRASTORNOS DEL SUEÑO}

Los trastornos del sueño en MPS se pueden deber a la presencia de apneas obstructivas, hipopneas o apneas centrales ${ }^{(20)}$. También existen trastornos de regulación del sueño principalmente en las MPS tipo III. La presencia de apneas obstructivas del sueño es un hecho frecuente en las MPS tipo I, II, IV y VI y se asocia a una elevada morbimortalidad (2). Las apneas obstructivas de causa multifactorial se deben a los depósitos de GAGS (macroglosia, engrosamiento de tejidos blandos, hipertrofia amigdaliana, palatina y faríngea, reducción de la permeabilidad nasal, rinitis crónica y secreciones viscosas). Se suma a lo anterior la presencia de anomalías faciales, que en conjunto llevan al colapso de la via aérea superior. Un estudio del grupo de Jhon y colaboradores, mostró que $85 \%$ de los pacientes con MPS presentaba algún grado de apnea obstructiva del sueño (AOS) sin existir alguna relación entre los aspectos clínicos sugerentes de apnea y los hallazgos del polisomnograma, sugiriendo que este estudio debe practicarse en forma precoz ${ }^{(24)}$. Otro estudio de Leighton y colaboradores involucró a 26 pacientes con MPS concluyó que la severidad de las AOS pueden estar asociadas con el tipo de MPS(21)

La enfermedad pulmonar restrictiva de los pacientes con 
MPS reduce aún mas el umbral de desaturación nocturna y retención de $\mathrm{CO}_{2}$. Las AOS conducen a un anormal ciclo de sueño-vigilia, con una reducción de los tiempos de sueño profundo y por ende potencial trastorno conductual durante el día. Las apneas de origen central en los pacientes con MPS se deben fundamentalmente a compresión de medula espinal, o por efecto de aumento de la presión intracraneana ya sea por hidrocefalia o por efecto a nivel de tronco encefálico(18)

El manejo de las AOS en los pacientes con MPS, comprende como primera etapa el manejo quirúrgico adenotonsilar, situación que es generalmente transitoria para posteriormente planificar el soporte ventilatorio dependiendo de la gravedad. En último término existe la traqueostomía (TQT) la cual debe ser siempre evaluada por un equipo multidisciplinario junto a los padres, considerando no sólo variables biológicas, sino biopsicociales (situación social, red de apoyo, habitabilidad de vivienda, educación de los padres). Las indicaciones de TQT son: obstrucción severa de vía aérea superior, no mejoría clínica con presión positiva de via aérea, estridor severo asociado a infiltración laríngea de GAGS, traqueobroncomalacia severa y obstrucción múltiple de la via aérea inferior ${ }^{(3-25)}$.

\section{CONSIDERACIONES ANESTÉSICAS}

Los pacientes con MPS requieren manejo anestésicos por múltiples causas a lo largo de su vida. Muchos pacientes son sometidos a instalación de catéter venosos central, fibrobroncoscopía, tomografía axial computada o resonancia magnética nuclear mediante intubación. Independiente de la gravedad de cada paciente o del tipo de MPS, el uso de anestésico incrementa el riesgo de morbilidad, pudiendo ser hasta $20 \%{ }^{(36)}$. Un estudio reciente, evaluó el riesgo anestésico en 17 pacientes con MPS, y mostró una incidencia global de intubación difícil de $25 \%{ }^{\left({ }^{(37)}\right.}$. La alta mortalidad perioperatoria de los pacientes con MPS sometidos a procedimientos, son debidas al compromiso respiratorio y cardiovascular. Algunos trabajos en relación a cuidados anestésicos en MPS tipo IV, demuestra riesgo de intubación, sugiriendo el uso de máscara laríngea, intubación con fibrobroncoscopía, uso de video laringoscopia (Glydscope) y el uso de anestesia regiona|(38).

Es fundamental, antes de cualquier intervención, descartar algún compromiso cardiovascular (alteraciones del ritmo, hipertensión pulmonar), ya que son las complicaciones cardiacas una de las principales causas de mortalidad perioperatoria. Es importante la correcta selección del tubo endotraqueal, considerando que generalmente requieren un tubo endotraqueal de menor tamaño, por el riesgo de estenosis subglótica o edema subglótico post cirugía. La extubación de aquellos pacientes bajo soporte ventilatorio debe ser programada y asistida considerando un horario hábil, diurno, con la disponibilidad de especialistas. En pocos casos, debe considerarse la TQT de urgencia, por lo que el contacto con otorrinolaringólogo debe ser expedita ${ }^{(39)}$.

Las complicaciones postoperatorias de los pacientes con MPS mas frecuentes involucran edema laríngeo, edema o inflamación subglótica, principalmente cuando ha estado asociado con una intubación dificultosa o varios intentos de intubación. Se describe complicaciones tardías, e incluso edema pulmonar post intubación, por lo que uno tiene que estar atento a la aparición de insuficiencia respiratoria aguda post-cirugía en pacientes con MPS(40).

\section{ROL DEL NEUMÓLOGO PEDIATRA}

El rol del especialista broncopulmonar está dado desde el momento de la sospecha diagnóstica, dado los problemas que muchos de estos pacientes presentan. Dependiendo del tipo de MPS, existen diversas manifestaciones pulmonares, siendo mas precoces en las MPS del tipo I, II y VI; mientras que las tipo III son mas tardías y asociadas principalmente con hipersecreción bronquial, defectos en el drenaje mucociliar, trastornos de deglución y aspiraciones pulmonares recurrentes. En las MPS tipo IV, el compromiso esquelético es la regla, pudiendo haber complicaciones de vía aérea debida a compresiones extrínsecas, deformaciones torácicas asociadas xifoescoliosis severa. En los pacientes que son capaces de cooperar, la evaluación de la función pulmonar debe realizarse periódicamente, al menos cada 6 meses, especialmente en aquellos que inician alguna terapia ya sea reemplazo enzimático o trasplante de medula ósea. La mejoría de la función pulmonar ha sido uno de los pilares fundamentales como respuesta al uso de estas terapias. Debe existir atención frente al eventual desarrollo de síntomas de obstrucción de la via aérea superior y aparición de apneas obstructivas del sueño, por lo que la necesidad de estudios durante el sueño es fundamental. La indicación de soporte ventilatorio no invasivo nocturno ha permitido que algunos niños normalicen sus estudios de oximetría y sueño.

\section{CONCLUSIÓN}

La MPS representa una enfermedad genética que involucra un gran desafío diagnóstico y terapéutico. Una vez establecido su diagnóstico, el manejo multidisciplinario es fundamental dado el amplio compromiso sistémico que ésta involucra. La morbimortalidad asociada es elevada y por ende, la intervención temprana es fundamental para alcanzar una adecuada calidad de vida.

\section{REFERENCIAS}

I. Muenzer J. Overview of the mucopolysaccharidoses. Rheumatology 20। I; 50: 4- 12.

2. Muhlebach M, Wooten W, Muenzer J. Respiratory manifestations in Mucopolysaccharidoses. Paediatr Respir Rev 2011; 12: 133-8.

3. Yeung A, Cowan M, Horn B, Rosbe K. Airway management in children with Mucopolysaccharidoses. Arch Otolaryngol Head Neck Surg 2009; 135: 73-9.

4. Giugliani R, Federhen A, Muñoz M, Vieira T, Artigalás $O$, Lapagesse L, et al. Mucopolysaccharidosis I, II, and VI: Brief review and guidelines for treatment. Genet Mol Biol 20 I0; 33: 589-604.

5. Wold S, Derkay C, Darrow D, Proud V. Role of the pediatric otolaryngologist in diagnosis and management of children with mucopolysaccharidoses. Int J Pediatr Otorhinolaryngol 2010; 74: 27-31. 
6. Muenzer J, Wraith J, Clarke L. Mucopolysaccharidosis I: Management and Treatment Guidelines. Pediatrics 2009; 123: 1929.

7. Giugliani R, Harmatz P, Wraith J. Management Guidelines for Mucopolysaccharidosis VI. Pediatrics 2007; 120: 405- I8.

8. Ashworth J, Biswas S, Wraith E, Lloyd Ch. Mucopolysaccharidoses and the Eye. Surv Ophthalmol 2006; 51: 1-17.

9. Morishita K, Petty R. Musculoskeletal manifestations of mucopolysaccharidoses. Rheumatology 20 I ; 50: 19-25.

10. White K. Orthopaedic aspects of mucopolysaccharidoses. Rheumatology 201 I; 50: 26-33.

II. Braunlin E, Harmatz P, Scarpa M, Furlanetto B, Kampmann C, Loehr JP, Ponder K, Roberts W, Rosenfeld H, Giugliani R. Cardiac disease in patients with mucopolysaccharidosis: presentation, diagnosis and management. J Inherit Metab Dis 20 I I; 34: I I8397.

12. Valayannopoulos $\mathrm{V}$, Wijburg F. Therapy for the mucopolysaccharidoses. Rheumatology 201 I; 50: 49-59.

13. Clarke L, Heppner J. Mucopolysaccharidosis Type I. Gene Reviews. http://www.ncbi.nlm.nih.gov/books/NBKI I 62 (2 de septiembre de 2012).

14. Scarpa M. Mucopolysaccharidosis Type II. Gene Reviews. http:// www.ncbi.nlm.nih.gov/books/NBKI274 (2 de septiembre de 2012).

15. Valstar M, Marchal JP, Grootenhuis M, Colland V, Wijburg F. Cognitive development in patients with Mucopolysaccharidosis type III (Sanfilippo syndrome). Orphanet J Rare Dis 20 I I; 6: 43-8.

16. Muenzer J. The Mucopolysaccharidoses: a heterogeneous group of disorders with variable pediatric presentations. J Pediatr 2004; 144: S27-S34.

17. Fundación Chilena de Pacientes con Enfermedades Lisosomales. www.felch.cl (3 de agosto de 20।2).

18. Santos S, López L, González L, Domínguez MJ. Hipoacusia y problemas de vía aérea en niños con mucopolisacaridosis. Acta Otorrinolaringol Esp 201 1; 62: 41 I-7.

19. Motamed M, Thorne, S, Narula A. Treatment of otitis media with effusion in children with mucopolysaccharidoses. Int J Pediatr Otorhinolaryngol 2000; 53: $121-4$.

20. Dempsey J, Veasey S, Morgan B, O'Donnell C. Pathophysiology of Sleep Apnea. Physiol Rev 20 10; 90: 47-II2.

21. Leighton S, Papsin B, Vellodi A, Dinwiddie D, Lane R. Disordered breathing during sleep in patients with mucopolysaccharidoses. Int J Pediatr Otorhinolaryngol 200 I; 58: 127-38.

22. Shih S, Lee J, Lin S, Sheu Y, Blickman J. Airway changes in children with mucopolysaccharidosis. Acta Radiologica 2002; 43: 40-3.

23. Nagano R, Takizawa Sh, Hayama N, Umemura Sh, Uesugi T, Nakagawa $\mathrm{S}$, et al. Three-dimensional CT and histopathological findings of airway Malacia in Hunter Syndrome. J Exp Clin Med 2007; 32: 59-61.

24. John Â, Fagondes S, Schwartz I, Azevedo AC, Barrios P, Dalcin $P$, et al. Sleep abnormalities in untreated patients with mucopolysaccharidosis type VI. Am J Med Genet 20 I ; I 55: I54651.

25. Jeong HS, Cho DY, Ahn KM, Jin DY. Complications of tracheotomy in patients with mucopolysaccharidoses type II (Hunter syndrome). Int J Pediatr Otorhinolaryngol 2006; 70: 1765-9.

26. Ingelmo PM, Parini R, Grimaldi M, Mauri F, Romagnoli M,
Tagliabue G, et al. Multidetector computed tomography (MDCT) for preoperative airway assessment in children with mucopolysaccharidoses. Minerva Anestesiol 201 I; 77: 774-80.

27. Kamin W. Diagnosis and management of respiratory involvement in Hunter syndrome. Acta Pædiatrica 2008; 97; 57-60.

28. Hope E, Farebrother M, Bainbridge D. Some aspects of respiratory function in three siblings with Morquio-Brailsford disease. Thorax 1969; 28: 335-41.

29. Harmatz P, Yu ZF, Giugliani R, Schwartz IV, Guffon N, et al. Enzyme replacement therapy for mucopolysaccharidosis $\mathrm{VI}$ : evaluation of long-term pulmonary function in patients treated with recombinant human N-acetylgalactosamine 4-sulfatase. J Inherit Metab Dis 20I0; 33: 5I-60.

30. Kirkpatrick K, Ellwood J, Walker R. Mucopolysaccharidosis type I (Hurler syndrome) and anesthesia: the impact of bone marrow transplantation, enzyme replacement therapy, and fiberoptic intubation on airway management. Pediatr Anesth 2012 (22): 74551.

31. Davitt S, Hatrick A, Sabharwal T, Pearce A, Gleeson M, Adam A. Tracheobronchial stent insertions in the management of major airway obstruction in a patient with Hunter syndrome (type-II mucopolysaccharidosis). Eur Radiol 2002; 12: 458-62.

32. Rodríguez M, Mackenzie W, Ditro C, Miller T, Chidekel A, Shaffer T. Skeletal Dysplasias: Evaluation With Impulse Oscillometry and Thoracoabdominal Motion Analysis. Pediatr Pulmonol 2010; 45: 679-86.

33. Wraith J, Clarke L, Beck M, Kolodny E, Pastores G, Muenzer J, et al. Enzyme replacement therapy for mucopolysaccharidosis I: a randomized, double-blinded, placebo-controlled, multinational study of recombinant human a-l-iduronidase (laronidase). J Pediatr 2004; 144: 581-8.

34. Wraith J. Enzyme replacement therapy with idursulfase in patients with mucopolysaccharidosis type II. Acta Pædiatr 2008; 97: 76-8.

35. Harmatz P, Ketteridge D, Giugliani R, Guffon G, Teles E, Miranda $M$, et al. Direct Comparison of Measures of Endurance, Mobility, and Joint Function During Enzyme-Replacement Therapy of Mucopolysaccharidosis VI (Maroteaux-Lamy Syndrome): Results After 48 Weeks in a Phase 2 Open-Label Clinical Study of Recombinant Human N-Acetylgalactosamine 4-Sulfatase. Pediatrics 2005; I 15: e68।-9.

36. Arna P, Whitley Ch, Wraith J, Webba H, Underhill L, Rangachari $\mathrm{L}$, et al. High rate of postoperative mortality in patients with mucopolysaccharidosis I: findings from the MPS I Registry. J Pediatr Surg 2012; 47: 477-84.

37. Frawley G, Fuenzalida D, Donath S, Yaplito-Lee J, Peters H. A retrospective audit of anesthetic techniques and complications in children with mucopolysaccharidoses. Pediatr Anesth 2012; 22: 737-44.

38. Theroux M, Nerker T, Ditro C, Mackenzie W. Anesthetic care and perioperative complications of children with Morquio síndrome. Pediatr Anesth 2012; 22: 901-7.

39. Shinhar Sh, Zablocki H, Madgy D. Airway Management in Mucopolysaccharide Storage Disorders. Arch Otolaryngol Head Neck Surg 2004; 130: 233-7.

40. Walker R, Colovic V, Robinson D, Dearlove O. Postobstructive Pulmonary Oedema During Anaesthesia In Children With Mucopolysaccharidoses. Paediatr Anaesth 2003; 13: 44I-7. 